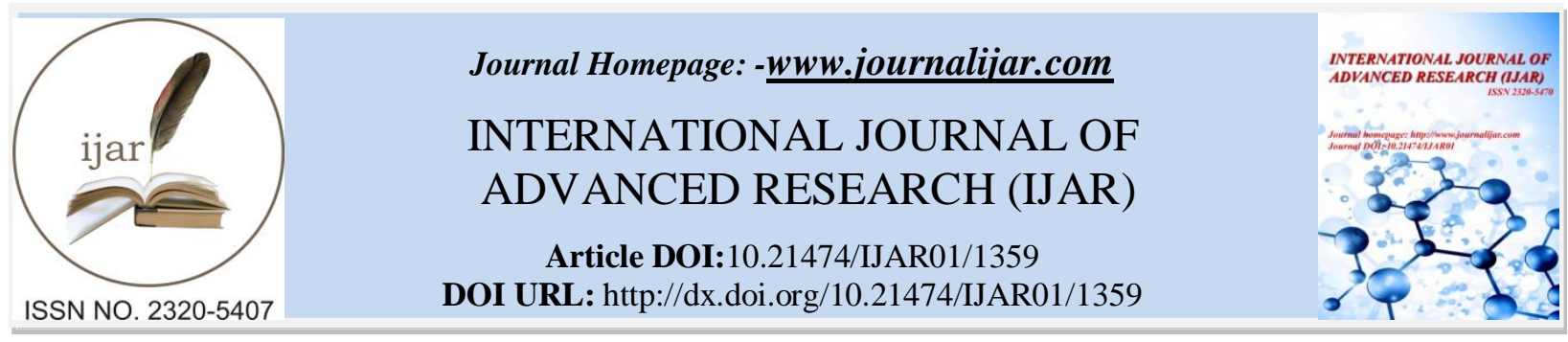

RESEARCH ARTICLE

\title{
Measurement of Liver Size and Factors Affecting it by Using CT or Ultrasonography
}

Zakaria Ali Mousbah Ahmad and Prof. Dr sci. med. Sanja Stojanovic

Department of Radiology, Faculty of Medicine, University of Novi Sad, 21000 Novi Sad, Serbia

\section{Manuscript Info}

Manuscript History

Received: 12 June 2016

Final Accepted: 12 July 2016

Published: August 2016

Key words:

Liver Size, factors, computer

tomography, ultrasonography

\section{Abstract}

Aim. We sought to determine the size and Factors Effecting it by Using CT or Ultrasonography. Methods. Review used the descriptivenormative research design in recording, describing, interpreting, analyzing and comparing updated data from authoritative sources. Data were evaluated descriptively. The influence of multiple variables on liver size was assessed by means of a covariance analysis. Results. The ten sources found, analyzed, compared and recommended the use of different factors, which effect liver size and span. These are gender, age, BMI, and body surface area and various methods, such as CT and PET scans, ultrasound and anthropometrical equipment. Taking into account the vital role of the liver in ensuring the continued survival on the human body, this has resulted in various attempts to study more about the organ in question and to examine its various functions. Using the descriptive-normative approach, the researchers for this study were able to examine the following findings pertaining to factors affecting liver size. First, the primary factor identified by the various studies as having potential influence on liver size and volume was biological sex, which was also cited as having a similar influence on standard uptake value (SUV). To a lesser extent, it was also noted that age and body mass had observable effects on liver size. Liver span, meanwhile, was noted as being best predicted by height and body surface area, while BMI and height were noted as predictors of liver diameter. Conclusion: The Liver volume assessed with CT scanning has correlated well with age reciprocally and with body height positively. and ultrasonography are currently considered the gold standard methods to determine liver size, both techniques using volume as the measure of liver size. Liver volume is a better way to assess liver size as it represents the entire liver, rather than linear measurements in single planes.

Copy Right, IJAR, 2016,. All rights reserved.

\section{Introduction:-}

The liver is an essential organ, which performs critical functions ((Sharma \&Priyanka 2015). These include digestion, metabolism, nutrient storage, and immunity. The liver has the marvelous capability of rejuvenating dead or injured body tissues and growing rapidly to regain its normal size and original functions. Measuring liver volumes, particularly by $\mathrm{CT}$, will be useful to radiologists in that CT has an extensive set of segmentation tools that 
can assess the whole liver, This present study used CT and a liver analysis software in determining the factors, which influence or affect the size of the liver (Sharma \&Priyanka, 2015).

\section{Methodology:-}

This review used the descriptive-normative research design in recording, describing, interpreting, analyzing and comparing updated data from authoritative sources.

\section{Literature Review:-}

\section{Estimated Factors through CT Software:-}

In the pursuit of the factors, which effect the volume of the liver, a research pair (Sharma \&Priyanda 2015), conducted a cross-section study through a sample of volunteers from the Kasturba Medical Hospital and College in Manipal, India. The researchers gathered the assistance and records of 124 volunteer male patients and 124 volunteer female patients who sought CT scan services at the hospital for various reasons. The volunteers' ages ranged from 20 to 80 . The researchers used abdomen helical protocol and reconstructed the raw data obtained. They also used the Mann Whitney $U$ test in exploring the average difference in the volume of liver between two groups, one aged 20 to 50, and the other aged 51 to 80. Of the four assumed factors of age, sex, body size and shape, the study found that gender is the only factor, which significantly affects the size of the liver and slightly the volume. The study found that the other assumed factors of age, body size and shape did not influence either the size of the volume (Sharma \&Priyanda 2015).

Earlier studies conducted in 2007 suggested a slight negative correlation between total volume and age (Sharma \&Priyanda 2015). Another study conducted by Chan Se and his team in 2006 also found that liver volume is genderdependent, with the male liver slightly heavier than that of the female even if they have the same body weight. Still another earlier study conducted in 2003, which used ultrasonography, concurred on gender as a significant determinant of liver size between males and females at $14.5 \mathrm{~cm}$ as against 13,5 as an absolute difference. This present study made a similar conclusion. At the same time, this study demonstrated the important role of CT and other post processing software in estimating these factors (Sharman \&Priyanda 2015).

\section{The effect of Age and BMI on Liver Size:-}

Recent cross-sectional study, which explored the impact of anthropometrical parameters on portal vein diameter and liver size together through the use of ultrasound on volunteers from Karachi, Pakistan, came out with a different finding (Siddiqui 2014). The study drew its findings from the results of gray scale ultrasonography applied on 459 assumed healthy volunteers. Its findings showed that both liver size and portal vein diameter increased with age. Liver size also increased with a rise in body mass index. These findings posed a sharp difference from the earlier study and findings that gender did not assert any influence in liver size or the portal vein diameter. It thus concluded on the reliability of age and body mass index in preventing false diagnoses of an enlarged liver. At the same time, it provided evidence of the dependability of anthropometrical measurements in helping clinicians choose the right recipients of liver transplants (Siddiqui 2014).

A large survey conducted by Kratzer and his team also concluded that age strongly influenced hepatic span as this present study did (Siddiqui 2014). Udoh and his team presented another sustaining conclusion, which suggested that liver size increases with age. The increase is believed to be linked to workload and the corresponding metabolic rise of advancing age (Siddiqui 2014).

The present study sustains previous experiments that age and body mass index physically affect liver span and portal vein diameter (Siddiqui 2014). Its finding can also help in the diagnosis of NAFLD and portal hypertension as well as routine radiological operations (Siddiqui 2014).

Hepatic Variations in SUV in PETR-CT Studies (Batalles et al. 2013)

A retrospective study endeavored to uncover the standard uptake value or SUV changes that occur to the body mass index of patients after a full body PRT-CT scans (Batalles et al. 2013). A sample of 603 patient volunteers was secure, 305 of whom were women, and 298 were men. It analyzed their ages, blood glucose levels, height and weight and the dose of 18FFDG and calculated the SUV mean value in connection with gender and body mass index or BMI. Analyses found that mean SUV was substantially higher in men than in women. It also went higher in both genders whose weight increased, and more so with obese ones. The study found that gender, age, and BMI were the best predictors of SUV measurements (Batalles et al. 2013). 
Results of this study suggest that the use of physiological hepatic metabolic activity as the reference for abdominal and pelvic deposits was unreliable at least in overweight and obese male patients (Batalles et al. 2013).

\section{Reliability of ARFI Method of Liver Stiffness Quantification:-}

Three expert operators examined ten healthy volunteers to determine and evaluate the inter- and intra-observer variability of acoustic radiation force impulse of AFFI quantification in certain parts of the liver in terms of age, body mass index, and liver-capsule-to-box distance. (Jaffer et al. 2012). The experts conducted the assessment at an interval of a week from three segments of the liver. They applied the Cronbach s statistics in the reliability analysis of inter- and intra-observer analysis (Jaffer et al. 2012).

The experts found ARFI to be a reliable method of quantifying liver stiffness and also capable being reproduced if taken from two of the three segments (Jaffer et al. 2012). The third segment, Segment 3 ARFI, proved unreliable. The experts, thus, recommended that it be avoided in an analysis. They also found greater accuracy in measurements taken at the greatest allowable CB distance (Jaffer et al. 2012).

\section{Clinical vs. Ultrasound Evaluation of Liver Size:-}

Clinicians use various techniques in measuring liver volume (Silva et al. 2010). These include radiography, scintigraphy, computer tomography or CT, magnetic resonance imaging or MRI, and ultrasonography. The last is the initial imaging method of choice for evaluating liver conditions. It is most popular for important advantages over the others. These are its lower cost, quick use, non-invasiveness, and the absence of ionizing radiation, risks, and sedation contents and effects. These features hasten its technical application, particularly when used on children (Silva et al. 2010).

Some experts also agree that body weight is a factor to, or influences, liver size (Silva et al. 2010). Based on this concurring opinion, the present cross-sectional study looked into the comparative correlation of clinical and ultrasound methods in determining liver size based on body weight. It obtained the cooperation of 81 adult volunteers, 43 female and 38 males, from the NossaSenhora da Conceicao Hospital Diagnostic Center in Tubarao, Santa Catarina in Brazil. The research team conducted the examination between August 2008 and April 2009. In that period, they took two percussion measurements and used ultrasonography in the percussion site. The volunteers' mean BMI was 23 and 19 of them had less than 25 BMI or 23.5\% (Silva et al. 2010).

This study found that clinical examination of liver measurement correlated better with the use of ultrasound method (Silva et al. 2010). But the statistically significant difference between the obtained final means of the two methods presents a different or underestimated size of the actual adult liver samples. It also found that the volunteers with bigger body mass index, or those with greater than $25 \mathrm{BMI}$, registered an unsatisfactory result. The result was satisfactory with those with BMI less than 25. It suggested that the clinical method was a less reliable method to use on patients of this BMI range (Silva et al. 2010).

\section{Single Linear Hepatic Measurement Reliability:-}

Comprehensive studies on radionuclide have appraised methods affecting liver size, such as limitations set by poor resolution, body habitus, field of view, breathing movements, unsatisfactory acoustic window, and assessor's dependency. Researches presented the reliability of sonographic measurement of the right MCL plane as indicating liver size. What still needed to uncover was the set of criteria in setting liver size through linear measurements by CT or MRI. And measuring hepatic volume takes up much time and requires a given software. A busy clinical radiologist, therefore, will prefer a simplified but reliable measuring tool or approach in determining liver size from comparative studies (Verma et al. 2013).

This present study was based on, and motivated by, four separate linear liver measurements assessed as liver size indicators and described by Gosink and Leymaster (Verma et al. 2013). It first chose between MHP CC and Max CC as to which would be easier to measure. The researchers chose Max CC, although it could be less reliable when used in the presence of Reidel's lobe at the right lobe of the liver. Max CC was determined to be comparable as reliability in indicating liver size as MHP CC in this current study. It took note of the suggested criterion made by Kratzer and his team that liver length greater than $16 \mathrm{~cm}$ should indicate hepatomegaly as sustained by their earlier study. Another recent study came up with results similar to this present study about hepatic volumes of normal and healthy livers (Verma et al. 2010). 
The objective of the present study intends to offer the busy radiologic practitioner with a reliable and practical method for obtaining liver size (Verma et al. 2010). It is also easy to access in that these linear measurements can be secured from any image archiving and communication system. Any such system does not require image processing or a specific 3D workstation and serial studies of the same patient can be easily compared (Vermaet al. 2010).

This study endorses the validity of single linear measurements of liver size and the reliability of Max CC and MHP AP and their products in indicating the liver size and in comparing liver sizes in serial studies (Verma et al. 2010). Both Max CC and MHP correlate in similar ways with hepatic volume. It also found Max CC measurement of the liver size and MHP CC hepatic dimensions as practicable and easy for routine applications. Moreover, linear hepatic measurements and their products may be used with confidence in determining liver size on MRI (Verma et al. 2010).

Liver Span and Factors through Ultrasound Measurement

This prospective study aimed at setting up a normal figure for a liver span, its relationships with anthropometric factors and establishing a standard ultrasound measurement of liver span (Tarawneh et al. 2009). These anthropometric factors include age, gender, body weight, body height, BMI and body surface. The study was specifically intended for adult volunteers in Jordan, where the ultrasound was not too well known (Tarawneh et al. 2009).

Clinical evaluation of liver size by percussion and palpation proved to be an unreliable and inaccurate basis (Tarawneh et al. 2009). Yet it is most frequently requested by clinicians for the evaluation of liver size. This study was conducted with 517 volunteer patients at the Jordan University Hospital between March 2007 and April 2008. The volunteers were 242 male and 275 female whose ages ranged from 18 to 76 . Statistical analyses on the secured data were conducted in determining the relationship between liver span and the anthropometric factors mentioned (Tarawneh et al. 2009).

Findings of the study showed a high-level and significant contribution of all the factors to the liver span of female volunteers. The same finding was obtained for male volunteers except for BMI. Specifically, the best predictor of liver span was height among the male volunteers and body surface area among the female volunteers. Height and body surface area were also reliable indicators in both genders and were concluded as the best determinants of liver span (Tarawneh et al. 2009).

\section{Liver Volume with Spiral CT:-}

This study sought the normal liver volume of adult north Indian volunteers by the use of spiral CT (Agraval et al. 2009). The initial assumption was the reciprocal correlation of age and body height as positively correlating with liver volume. The specific advantages of CT made it the choice method for the assessment.As bolstered by earlier findings, this present study argues for the relationship between liver volumes and age and body height. The liver volume also highly correlates with age, body height and weight, BMI and BSA but not age. Liver volume is, thus, found to be a reliable indicator of liver size as well as measurement of liver volume when spiral T is used. The study concludes that spiral CT is a practical, fast and easy measurement equipment of liver volume in the clinical setting (Agraval et al. 2009).

\section{Standard Liver Volume:-}

The recent rise in demand for liver transplant donations urged for accurate means of calculating and establishing the standard liver volume of SLV according to recipient's body indices as important criteria (Chandramohan et al. 2007). These researchers used three formulas obtained from authoritative literature on Western and Japanese sample populations. The researchers measured the total volume or TLV of 238 volunteer patients in the Indian population by using axial helical CT images. The volunteers' body surface area was computed from their height and weight. And CT measurements were compared with the SLV derived from the formulas (Chandromohan et al. 2007).

The study revealed the lack of suitableness of the formulas' measurements used on the Japanese population samples as compared with the present Indian population samples (Chandramohan et al. 2007). Western formulas and the Japanese formula underestimated the Indian patients' SLV. Indian BSA came between a higher Western BSZ and a lower Japanese BSA. The difference could be the large number of children in the Japanese study. Earlier studies revealed that CT volume measurements were closely similar to the actual liver volume at a high $95 \%$. Current researchers adapted certain measures to correct the errors. As a result, this present study confirmed and concluded 
the superficial correlation between age and TLV and the lack of significant effect of gender on SLV calculation (Chamdramohan et al. 2007).

Of the three formulas used for the experiment, the one based on body surface area and used on Western population samples resembled the liver volume estimates of the Indian population sample, with only a slight overestimation (Chandramohan et al. 2007. But it should not be used in calculating for SLV in cases of extreme BSA (Chandramohan et al. 2007).

\section{Factors of Liver Size:-}

This sonographic survey aimed at determining liver size from a random population sample in fixing normal standards and searching as well as studying influencing factors of liver size (Kratzer et al. 2003). The sample popular consisted of 2,080 subjects, 983 male, and 1,097 female, aged 18 to 88, from a rural community in Southwestern Germany. The volunteers were asked to submit to ultrasound examination, physical examination and interviews on the probable influencing factors. Descriptive data evaluation was used with covariance analysis of the variables. The study found that the sonographic measurement method was an easy and practical method that clinicians can use routinely. It concluded that body mass index or BMI and body height as the most important influencing factors of liver diameter when measured at the midclavicular line.. The study suggested a liver diameter of $16 \mathrm{~cm}$ or more in the right MCL be considered in cases of an enlarged liver. BMI and height must also be considered with care in borderline cases (Kratzer et al. 2003).

Analysis

Sharman \&Priyanda (2015) directly pointed to gender as a significant factor affecting liver size, using CT and other processing software. Siddiqui (2014) argued for age and BMI as affecting the liver size and by using anthropometrical measurements as tools. Battales and his team (2013) likewise identified BMI as influencing liver size through the use of PET-CT scanning. Silva and team (2010) recommended the use of ultrasound in determining liver size but not with patients with high BMI. Verma and team (2010), on the other hand, recommended the use of Max CC and MHP CC as reliable formulas in establishing liver size. Tarawneh and team (2009) identified height and body surface as the best predictors of a liver span. Agrawal and team (2009) pushed for the use of spiral CT in measuring liver volume and size. And Chandramohan and team (2007) also named body surface area as a reliable indicator of liver size but in specific populations. Only Jaffer and team (2012) tested and suggested the use ofARFI as a reliable method for quantifying liver stiffness in two out of three liver segments.

\section{Conclusion:-}

The ten sources found, analyzed, compared and recommended the use of different factors, which effect liver size and span. These are gender, age, BMI, and body surface area and various methods, such as CT and PET scans, ultrasound and anthropometrical equipment. Taking into account the vital role of the liver in ensuring the continued survival on the human body, this has resulted in various attempts to study more about the organ in question and to examine its various functions. Using the descriptive-normative approach, the researchers for this study were able to examine the following findings pertaining to factors affecting liver size. First, the primary factor identified by the various studies as having potential influence on liver size and volume was biological sex, which was also cited as having a similar influence on standard uptake value (SUV). To a lesser extent, it was also noted that age and body mass had observable effects on liver size. Liver span, meanwhile, was noted as being best predicted by height and body surface area, while BMI and height were noted as predictors of liver diameter.

With respect to measurement methods, ARFI was noted for its effectiveness and reliability in quantifying liver stiffness. Moreover, the use of ultrasound technology was described as being highly useful in a clinical setting, but that usage on patients with BMI over 25 may yield inaccurate readings. Meanwhile, hepatic volume could be determined by using Max CC to measure liver size, while using MHP CC for hepatic dimensions. Combined with the use of MRI, these methods become capable of yielding much more accurate findings. Another useful approach in this regard is the spiral CT method, which was cited for practicality, efficiency and ease of use.

In light of the aforementioned findings, the following recommendations can be made. First, the most useful variables in measuring the liver's dimensions and overall size can be identified as gender, BMI, height and age. Meanwhile, evaluating the methods mentioned above will indicate the notable effectiveness of the Max CC and MHP CC formulas, as well as the ultrasound method in the case of patients with a BMI value below 25. For the most 
comprehensive results, the clinician is advised to consider these particular variables and methods, but to be ready to account for other factors and employ alternative methods as the situation may require.

\section{References:-}

1. D Agrawal, R Lalwani, A Asghar, A Sahai, P Sharma, R Singh. (2009) Assessment of Liver Volume with Spiral Computerized Tomography scanning in North Indian Adults. The Internet Journal of Radiology. Volume 13 (1).

2. Batallés, S. M., Villavicencio, R. L., Quaranta, A., Burgos, L., Trezzo, S., Staffieri, R., \& Pezzotto, S. M. (2013). Variations of the hepatic SUV in relation to the body mass index in whole body PET-CT studies. Revista Española de Medicina Nuclear e Imagen Molecular (English Edition), 32(1), 26-32.

3. Chandramohan, A., Eapen, A., Govil, S., Govil, S., \&Jeyaseelan, V. (2007). Determining standard liver volume: assessment of existing formulae in Indian population. Indian Journal of Gastroenterology, 26(1), 22.

4. Jaffer, O.S., Lung, P.F.C., Bosanac, D., Patel, V.M., Ryan, S.M., Heneghan, M.A., Quaglia, A. and Sidhu, P.S. (2014). Acoustic radiation force impulse quantification: repeatability of measurements in selected liver segments and influence of age, body mass index and liver capsule-to-box distance. The British journal of radiology.

5. Matthews, K. A., Meilahn, E., Kuller, L. H., Kelsey, S. F., Caggiula, A. W., \& Wing, R. R. (1989). Menopause and risk factors for coronary heart disease. New England Journal of Medicine, 321(10), 641-646.

6. Sharma, D. (2015). Estimation of Factors Effecting Volume of Liver using Liver Analysis Software in Computed Tomography. International Journal of Pharma and Bio Sciences, 6(4), 792-798.

7. Da Silva, R. R. M., Rodrigo, P. B., \&Siqueira, M. V. (2010). Correlation between clinical evaluation of liver size versus ultrasonography evaluation according to body mass index (bmi) and biotypes. Rev Med Chile, 138, 1495-1501.

8. Siddiqui, T. R., Hassan, N., \& Gul, P. (2014). Impact of anthropometrical parameters on portal vein diameter and liver size in a subset of Karachi based population. Pakistan journal of medical sciences, 30(2), 384.

9. Tarawneh, E. S., Hadidy, A. M., Haroun, A. A., Mahafza, W. S., Samara, O. A., Arafeh, F. M., \&Alsharif, A. A. (2010). Ultrasound Measurement of Liver Span in Jordanian Adults: A Preliminary Experience. Jordan Medical Journal,43(3).

10. Verma, S. K., McClure, K., Parker, L., Mitchell, D. G., Verma, M., \& Bergin, D. (2010). Simple linear measurements of the normal liver: interobserver agreement and correlation with hepatic volume on MRI. 